This article is published with open access at Springerlink.com

\title{
On positive commutative tomonoids
}

\section{Thomas VetTERLEIN}

\begin{abstract}
We discuss totally ordered monoids (or tomonoids, for short) that are commutative, positive, and finitely generated. Tomonoids of this kind correspond to certain preorders on free commutative monoids. In analogy to positive cones of totally ordered groups, we introduce direction cones to describe the preorders in question and we establish between both notions a Galois connection. In particular, we show that any finitely generated positive commutative tomonoid is the quotient of a tomonoid arising from a direction cone. We furthermore have a closer look at formally integral tomonoids and at nilpotent tomonoids. In the latter case, we modify our approach in order to obtain a description that is based on purely finitary means.
\end{abstract}

\section{Introduction}

An interest in totally ordered monoids, or tomonoids as we say in accordance with [4], is present in diverse fields. For instance, in computational mathematics and in particular in connection with Gröbner bases of polynomial ideals, compatible, positive total orders on an $\mathbb{N}^{n}$, called monomial or term orders, play an important role; see, e.g., [2]. An early overview of the extensive research on totally ordered semigroups can be found in [6]; a comprehensive treatment of more recent times is [4].

Our own motivation to study tomonoids is related to a comparably young research area. Namely, these structures are of central significance in fuzzy logic [8]. The primary connective of propositional fuzzy logic is the conjunction, which is usually interpreted by a t-norm [11]. A t-norm is a binary operation that makes the real unit interval, endowed with its natural order, into a tomonoid. Tomonoids arising in this way have the additional properties of being commutative and negative, where "negative" is the same as "positive" in the dual picture adopted here. Accordingly, we focus in this paper on positive, commutative tomonoids, and we will furthermore generally assume that the tomonoids are, as monoids, finitely generated.

We note in addition that the generalised semantics of fuzzy logics often involve MTL-algebras or their subclasses. In particular, the fuzzy logic with the same name corresponds to MTL-algebras. The variety of MTL-algebras is

Presented by S. Pulmannova.

Received May 8, 2014; accepted in final form January 16, 2015.

2010 Mathematics Subject Classification: Primary: 06F05; Secondary: 03G25, 03B52.

Key words and phrases: totally ordered monoid, direction cone.

This research was partially supported by the Austrian Science Fund (FWF): project I $1923-\mathrm{N} 25$. 
in turn generated by its finite totally ordered members [1]. But finite MTLchains are nothing but finite negative commutative tomonoids endowed with an implication as an additional operation. The implication operation is definable from the remaining structure, so that finite MTL-chains can actually be considered as belonging to those structures that are studied in the present paper.

We guess that there are many approaches to the problem of analysing and describing finitely generated tomonoids. Here we make use of the wellestablished method of describing commutative monoids by means of congruences on free commutative monoids $[3,7]$. In our case, a compatible, positive total order needs to be taken into account additionally. To this end, we in fact do not directly consider the congruences in question, but instead the preorders induced by the total order of tomonoids on their associated free monoids. This approach is quite practical because the preorders "encode" both the congruence and the total order. We are, in this way, led to the question of how compatible, positive total preorders on an $\mathbb{N}^{n}$ can be characterised.

Compatible preorders on free monoids have been studied, e.g., in [10]. Here, our key idea is the following. Recall that the order of a partially ordered group is described by its positive cone. In analogy to this idea, we introduce what we call a direction cone, whose characteristic properties are quite similar to those of positive cones of partially ordered groups. The correspondence between the preorders in question and direction cones is many-to-one; that is, not all preorders on the $\mathbb{N}^{n}$ are represented by a direction cone. However, there is a Galois connection between the two notions, and each compatible, positive total preorder can be restricted to a preorder arising from a direction cone. Consequently, the class of tomonoids described by direction cones is rich enough that each finitely generated, positive, commutative tomonoid is a quotient of a member of this class.

The notion of a direction cone can be applied to some interesting subclasses. We consider here the conditions of formal integrality as well as nilpotency. In the latter case, we deal with finite structures, and it seems appropriate to describe them by finitary means. However, directions cones are usually infinite. Direction f-cones, in contrast, are finite; they are defined similarly to direction cones, but tailored to the case of nilpotency. We establish a Galois connection also for this more special situation and we show that any nilpotent finite, positive, commutative tomonoid is the quotient of a tomonoid induced by a direction f-cone.

The paper is organised as follows. We introduce in Section 2 the basic notions around tomonoids and establish their correspondence with preorders on $\mathbb{N}^{n}$. In a first step towards the description of such preorders, we consider in Section 3 a comparably well-understood subclass of tomonoids, namely those that arise from the positive cone of totally ordered Abelian groups. In an analogous manner, we then proceed in Section 4 with the case of finitely generated tomonoids and we establish their correspondence with direction cones. 
Formally integral tomonoids are dealt with in the Section 5. We then finally turn to nilpotent tomonoids. For this class of tomonoids, it makes sense to modify the approach developed so far; we do so in Section 6. Some general remarks in Section 7 conclude the paper.

\section{Totally ordered monoids}

We study the following structures in this paper.

Definition 2.1. An algebra $(L ;+, 0)$ is a monoid if $(\mathrm{i})+$ is associative and (ii) $a+0=0+a=a$ for any $a \in L$. A monoid $(L ;+, 0)$ is called commutative if + is commutative.

A partial order $\leqslant$ on a monoid $L$ is called compatible if, for any $a, b, c \in L$, $a \leqslant b$ implies $a+c \leqslant b+c$ and $c+a \leqslant c+b$. A structure $(L ; \leqslant,+, 0)$ such that $(L ;+, 0)$ is a monoid and $\leqslant$ is a compatible partial order on $L$ is a partially ordered monoid, or pomonoid for short. In case that the partial order is total, we refer to $L$ as a totally ordered monoid, or tomonoid for short. Finally, a pomonoid is commutative if so is its monoidal reduct.

Pomonoids are often written multiplicatively; the monoidal operation is then denoted by - or a similar symbol and the monoidal identity by 1 . We prefer to write $(L ;+, 0)$ rather than $(L ; \cdot, 1)$ because the emphasis of this article is on free commutative monoids, which are usually written additively.

We are interested in commutative tomonoids subject to the following conditions.

Definition 2.2. Let $(L ; \leqslant,+, 0)$ be a commutative pomonoid. $L$ is called positive if 0 is the bottom element.

Furthermore, $L$ is called finitely generated if $L$, as a monoid, is generated by finitely many elements.

Tomonoids considered in this paper are always positive, commutative, and finitely generated. We will abbreviate "positive commutative" by "p.c."

A tomonoid is trivial if it consists of the zero, the monoidal identity, alone. We will tacitly assume throughout this paper that all tomonoids are nontrivial. Moreover, a set of generators of a tomonoid $L$ will be understood to be a non-empty, finite set of non-zero elements that generate $L$ as a monoid.

P.c. tomonoids possess, in addition to the partial order included in their signature, a partial order that depends on the monoidal reduct alone.

Definition 2.3. Let $(L ;+, 0)$ be a commutative monoid. For $a, b \in L$, let

$$
a \leqslant \mathcal{H} b \text { if } a+c=b \text { for some } c \in L \text {; }
$$

then $\leqslant_{\mathcal{H}}$ is called Green's preorder. If $\leqslant_{\mathcal{H}}$ is a partial order, we refer to $\leqslant_{\mathcal{H}}$ as the natural order on $L$. 
Lemma 2.4. Let $(L ; \leqslant,+, 0)$ be a p.c. pomonoid. Then Green's preorder $\leqslant \mathcal{H}$ on $(L ;+, 0)$ is a compatible partial order, whose least element is 0 . In other words, also $(L ; \leqslant \mathcal{H},+, 0)$ is a p.c. pomonoid. Moreover, $\leqslant$ extends $\leqslant \mathcal{H}$.

We finally establish the notation as regards free commutative monoids, which will play a central role. We identify the free commutative monoid over an $n$-element set, where $n \geqslant 1$, with $\mathbb{N}^{n}$. The addition is pointwise and the identity is $\overline{0}=(0, \ldots, 0)$, the $n$-tuple consisting solely of 0 's. For $i=1, \ldots, n$, we denote the $i$-th unit vector of $\mathbb{N}^{n}$ by $u_{i}$, that is, we put $u_{i}=(0, \ldots, 0,1,0, \ldots, 0)$, " 1 " being at the $i$-th position. Then $U\left(\mathbb{N}^{n}\right)=$ $\left\{u_{1}, \ldots, u_{n}\right\}$ is a set of generators of $\mathbb{N}^{n}$.

For $\left(a_{1}, \ldots, a_{n}\right),\left(b_{1}, \ldots, b_{n}\right) \in \mathbb{N}^{n}$, we put

$$
\left(a_{1}, \ldots, a_{n}\right) \preccurlyeq\left(b_{1}, \ldots, b_{n}\right) \text { if } a_{1} \leqslant b_{1}, \ldots, a_{n} \leqslant b_{n} .
$$

Clearly, $₫$ is the natural order on $\left(\mathbb{N}^{n} ;+, \overline{0}\right)$. Endowed with $₫, \mathbb{N}^{n}$ becomes a p.c. pomonoid.

Congruences and quotients. We compile a few definitions and facts concerning the formation of quotients of pomonoids.

Let $L$ be a p.c. pomonoid. By a pomonoid congruence of $L$, we mean an equivalence relation $\sim$ on $L$ such that $(\mathrm{i}) \sim$ is a congruence of $L$ as a monoid and (ii) for any $a_{0}, \ldots, a_{k} \in L$,

$$
a_{0} \leqslant a_{1} \sim a_{2} \leqslant a_{3} \sim \cdots \leqslant a_{k-1} \sim a_{k} \leqslant a_{0}
$$

implies $a_{0} \sim \cdots \sim a_{k}$. In this case, we endow the quotient $\langle L\rangle_{\sim}$ with the smallest partial order such that $\langle a\rangle_{\sim} \leqslant\langle b\rangle_{\sim}$ if $a \leqslant b$, with the induced addition + , and with the constant $\langle 0\rangle_{\sim}$. The result is a p.c. pomonoid again and called a pomonoid quotient of $L$. If $\langle L\rangle_{\sim}$ is totally ordered, we speak about a tomonoid congruence, leading to a tomonoid quotient. Moreover, if the 0-class consists of 0 alone, that is, if $\langle 0\rangle_{\sim}=\{0\}$, we call the congruence $\sim$ as well as the quotient $\langle L\rangle \sim$ pure.

In case of a naturally ordered free commutative monoid, we can characterise the pomonoid congruences as monoid congruences whose classes are convex.

Lemma 2.5. An equivalence relation $\sim$ on $\mathbb{N}^{n}$ is a pomonoid congruence of $\left(\mathbb{N}^{n} ; \sharp,+, \overline{0}\right)$ if and only if $(\mathrm{i}) \sim$ is a congruence of the monoid $\left(\mathbb{N}^{n} ;+, \overline{0}\right)$ and (ii) all $\sim$-classes are convex with respect to $\sharp$. In this case, the partial order on $\left\langle\mathbb{N}^{n}\right\rangle_{\sim}$ is its natural order $\leqslant \mathcal{H}$.

Proof. Let $\sim$ be a pomonoid congruence on $\mathbb{N}^{n}$. Then $a \preccurlyeq b \leqslant c \sim a$ implies $a \sim b \sim c$; hence, the $\sim$-classes are convex.

Conversely, let $\sim$ be a congruence of the monoid $\left(\mathbb{N}^{n} ;+, \overline{0}\right)$ such that all classes are convex. For $a_{0}, \ldots, a_{k} \in \mathbb{N}^{n},(2.2)$ implies $a_{0}+c_{0}+c_{2}+\cdots+c_{k} \sim a_{0}$, where, for each $i=0,2, \ldots, k-2$, we have $a_{i}+c_{i}=a_{i+1}$ and $a_{k}+c_{k}=a_{0}$. We conclude that $a_{0}, a_{0}+c_{0}, \ldots, a_{0}+c_{0}+\cdots+c_{k}$, and hence all the $a_{i}$ 's are pairwise equivalent. 
Finally, $\left\langle\mathbb{N}^{n}\right\rangle_{\sim}$ is endowed with the smallest partial order $\leqslant$ such that we have $\langle a\rangle_{\sim} \leqslant\langle a+c\rangle_{\sim}$ for any $a, c \in \mathbb{N}^{n}$. It is not difficult to check that $\leqslant$ coincides with the natural order $\leqslant \mathcal{H}$ of $\left(\left\langle\mathbb{N}^{n}\right\rangle_{\sim} ;+,\{\overline{0}\}\right)$.

The construction of an arbitrary finitely generated p.c. tomonoid can be described in two steps as follows.

Proposition 2.6. Let $\sim$ be a congruence of the monoid $\left(\mathbb{N}^{n} ;+, \overline{0}\right)$ such that all classes are convex with respect to $\sharp$ and $\langle\overline{0}\rangle_{\sim}=\{\overline{0}\}$. Then $\sim$ is a pure pomonoid congruence of $\left(\mathbb{N}^{n} ; \sharp,+, \overline{0}\right)$ and the quotient $\left(\left\langle\mathbb{N}^{n}\right\rangle_{\sim} ; \leqslant \mathcal{H},+,\{\overline{0}\}\right)$ is a p.c. pomonoid which is generated by $\left\langle u_{1}\right\rangle_{\sim}, \ldots,\left\langle u_{n}\right\rangle_{\sim}$.

Let furthermore $\leqslant$ be a compatible total order on $\left\langle\mathbb{N}^{n}\right\rangle_{\sim}$ extending its natural order $\leqslant \mathcal{H}$. Then $\left(\left\langle\mathbb{N}^{n}\right\rangle_{\sim} ; \leqslant,+,\{\overline{0}\}\right)$ is a p.c. tomonoid.

Any finitely generated p.c. tomonoid is isomorphic to a tomonoid arising in this way.

Proof. The first part follows from Lemma 2.5. The second part is clear.

For the last part, let $(L ; \leqslant,+, 0)$ be a p.c. tomonoid. Then the monoid $(L ;+, 0)$ is isomorphic to a quotient $\left(\left\langle\mathbb{N}^{n}\right\rangle_{\sim} ;+,\langle\overline{0}\rangle_{\sim}\right)$ of $\mathbb{N}^{n}$, generated by the non-zero elements $\left\langle u_{1}\right\rangle_{\sim}, \ldots,\left\langle u_{n}\right\rangle_{\sim}$. Identifying $L$ and $\left\langle\mathbb{N}^{n}\right\rangle_{\sim}, \leqslant$ extends the natural order $\leqslant_{\mathcal{H}}$ on $\left\langle\mathbb{N}^{n}\right\rangle_{\sim}$, by Lemma 2.4 .

From this last fact, we conclude that the $\sim$-classes of $\mathbb{N}^{n}$ are convex with respect to $\sharp$. In particular, $\langle\overline{0}\rangle_{\sim}$ is convex and does not contain $u_{i}$ for any $i$; hence, $\langle\overline{0}\rangle_{\sim}=\{\overline{0}\}$.

The representation of a p.c. tomonoid according to Proposition 2.6 is certainly not unique. In fact, the indicated set of generators need not be minimal; the same element can, for instance, appear here twice. For finitely generated p.c. tomonoids, there is an easy way to choose a canonical representation, based on the unique minimal set of generators; see, e.g., [4]. However, in this paper we will not do so; we keep matters simpler when accepting the indeterminacy.

Monomial preorders. According to Proposition 2.6, the construction of tomonoids requires two steps: first, a quotient of the monoid $\mathbb{N}^{n}$ with convex classes is formed; second, the natural order is extended to a total order. It is straightforward, and turns out to be convenient, to combine both steps in one: by considering preorders on $\mathbb{N}^{n}$.

A preorder on a set $A$ is a reflexive and transitive binary relation $\preccurlyeq$ on $A$. In this case, we write $a \prec b$ if $a \preccurlyeq b$ but not $b \preccurlyeq a$. A preorder $\preccurlyeq$ is called total if, for any pair $a, b \in A$, either $a \preccurlyeq b$ or $b \preccurlyeq a$. We associate with its symmetrisation $\approx$, where $a \approx b$ if $a \preccurlyeq b$ and $b \preccurlyeq a$. The equivalence class of some $a$ with respect to $\approx$ is called a $\preccurlyeq$-class and is denoted by $\langle a\rangle_{\preccurlyeq}$. The quotient with respect to $\approx$ is denoted by $\langle A\rangle_{\preccurlyeq}$, and its induced partial order is denoted by $\preccurlyeq$ again.

A preorder $\preccurlyeq$ on a monoid $(L ;+, 0)$ is called compatible if $a \preccurlyeq b$ implies $a+c \preccurlyeq b+c$; and $\preccurlyeq$ is strictly positive, or simply positive, if $0 \prec a$ for all $a \neq 0$. 
Proposition 2.7. Let $\preccurlyeq$ be a compatible, positive total preorder on $\left(\mathbb{N}^{n} ;+, \overline{0}\right)$. Then $\approx$ is a monoid congruence whose $\preccurlyeq$-classes are convex and $\langle\overline{0}\rangle_{\preccurlyeq}=\{\overline{0}\}$. Moreover, $\left(\left\langle\mathbb{N}^{n}\right\rangle_{\preccurlyeq} ; \preccurlyeq,+,\{\overline{0}\}\right)$ is a p.c. tomonoid generated by $\left\langle u_{1}\right\rangle_{\preccurlyeq}, \ldots,\left\langle u_{n}\right\rangle_{\preccurlyeq}$.

Conversely, let $(L ; \leqslant,+, 0)$ be a p.c. tomonoid, and assume that the $n \geqslant 1$ elements $g_{1}, \ldots, g_{n} \in L \backslash\{0\}$ generate $L$. Let $\iota: \mathbb{N}^{n} \rightarrow L$ be the surjective monoid homomorphism determined by $\iota\left(u_{i}\right)=g_{i}$ for $i=1, \ldots, n$. Define

$$
a \preccurlyeq b \quad \text { if } \quad \iota(a) \leqslant \iota(b),
$$

for $a, b \in \mathbb{N}^{n}$. Then $\preccurlyeq i s$ a compatible, positive total preorder of $\mathbb{N}^{n}$, and $\iota$ induces an isomorphism between $\left(\left\langle\mathbb{N}^{n}\right\rangle_{\preccurlyeq} ; \preccurlyeq,+,\{\overline{0}\}\right)$ and $(L ; \leqslant,+, 0)$.

Proof. Let $\preccurlyeq$ be a compatible, positive total preorder on $\mathbb{N}^{n}$. Then, for $a, b, c, d \in \mathbb{N}^{n}, a \approx c$ and $b \approx d$ imply $a+b \approx c+d$ by the compatibility of $\preccurlyeq$; hence, $\approx$ is a monoid congruence. As $\preccurlyeq$ is also positive, $\preccurlyeq$ extends $\preccurlyeq$, and it follows that the $\preccurlyeq$-classes are convex. Again by the positivity, the $\preccurlyeq$-class of $\overline{0}$ consists of $\overline{0}$ alone.

As $\preccurlyeq$ is compatible, the partial order $\preccurlyeq$ induced on $\left\langle\mathbb{N}^{n}\right\rangle_{\preccurlyeq}$ is compatible as well; that is, $\left(\left\langle\mathbb{N}^{n}\right\rangle_{\preccurlyeq} ; \preccurlyeq,+,\langle\overline{0}\rangle_{\preccurlyeq}\right)$ is a commutative pomonoid. Since, for any $a, b \in \mathbb{N}^{n}, a \preccurlyeq b$ or $b \preccurlyeq a,\left\langle\mathbb{N}^{n}\right\rangle_{\preccurlyeq}$ is actually a tomonoid. Moreover, since $\overline{0} \prec a$ for any $a \in \mathbb{N}^{n} \backslash\{\overline{0}\},\left\langle\mathbb{N}^{n}\right\rangle_{\preccurlyeq}$ is a p.c. tomonoid which is generated by $\left\langle u_{1}\right\rangle_{\preccurlyeq}, \ldots,\left\langle u_{n}\right\rangle_{\preccurlyeq}$.

Conversely, let $(L ; \leqslant,+, 0)$ be a p.c. tomonoid and $g_{1}, \ldots, g_{n} \in L \backslash\{0\}$ generate $L$ as a monoid. Let furthermore $\iota: \mathbb{N}^{n} \rightarrow L$ be as indicated and let $\preccurlyeq$ be defined by (2.3). By construction, $\preccurlyeq$ is transitive and reflexive, that is, a preorder. Then $\preccurlyeq$ is compatible because $\leqslant$ is, and $\iota$ is a monoid homomorphism. Moreover, $\preccurlyeq$ is positive because $L$ is positive, and hence $\iota(a) \leqslant 0$ holds only if $a=\overline{0}$. Finally, for $a, b \in \mathbb{N}^{n}$, we have $a \approx b$ if and only if $a \preccurlyeq b$, and $b \preccurlyeq a$ if and only if $\iota(a)=\iota(b)$; hence, $\iota$ induces an isomorphism as claimed.

In analogy to the case of monomial orders on $\mathbb{N}^{n}[2]$, we call a compatible, positive total preorder a monomial preorder.

By Proposition 2.7, any monomial preorder $\preccurlyeq$ on $\mathbb{N}^{n}$ gives rise to a p.c. tomonoid $L$, generated by $n$ elements. We refer to $L$ then as the tomonoid represented by $\preccurlyeq$.

Proposition 2.7 also states that, up to isomorphism, any finitely generated p.c. tomonoid $L$ arises in this way from a monomial preorder. In other words, describing finitely generated p.c. tomonoids can be done by describing monomial preorders. We do this here, using the following lemma.

Lemma 2.8. Let the monomial preorder $\preccurlyeq$ on $\mathbb{N}^{n}$ represent the tomonoid $L$. Then any pure tomonoid quotient of $L$ is represented by a monomial preorder extending $\preccurlyeq$. Conversely, any monomial preorder on $\mathbb{N}^{n}$ extending $\preccurlyeq$ represents a pure tomonoid quotient of $L$. 


\section{Tomonoids from totally ordered Abelian groups}

As a preparation of what follows, we review in this section a structure that is well known in the present context. Our topic is positive, commutative totally ordered monoids; a typical example is the positive cone of a totally ordered Abelian group. The latter is, as a monoid, in general not finitely generated even if the group is. As we are interested in finitely generated tomonoids, we will actually consider submonoids of the positive cone of a totally ordered group, which we just assume to generate the whole group. For basic facts on partially ordered groups, we refer to [5].

Definition 3.1. Let $(G ; \leqslant,+, 0)$ be a totally ordered Abelian group and assume that $G$ is generated by the $n \geqslant 1$ elements $g_{1}, \ldots, g_{n} \in G^{+} \backslash\{0\}$. Let $L$ be the submonoid of $G$ generated by $g_{1}, \ldots, g_{n}$ and let $L$ be endowed with the total order inherited from $G$, with the group addition, and with the constant 0 . Then we call $(L ; \leqslant,+, 0)$ a group cone tomonoid.

It is clear that a group cone tomonoid is a finitely generated p.c. tomonoid. It is furthermore easily seen how group cone tomonoids are characterised. We call a p.c. tomonoid $L$ cancellative if, for all $a, b, c \in L, a+c=b+c$ implies $a=b$. Note that in this case, for all $a, b, c \in L, a \leqslant b$ is equivalent to $a+c \leqslant b+c$.

Proposition 3.2. A finitely generated p.c. tomonoid $(L ; \leqslant,+, 0)$ is a group cone tomonoid if and only if $L$ is cancellative.

Proof. The "only if" part follows from the construction of a group cone tomonoid.

To see the "if" part, let $L$ be cancellative. Let $G$ be the group consisting of the differences of elements of $L$; see, e.g., [5, Chapter II.2]. Viewing $L$ as a subset of $G$, we introduce a total order on $G$ as follows: for $a, b, c, d \in L$, we define $a-b \leqslant c-d$ if $a+d \leqslant b+c$ in $L$. Then $(G ; \leqslant,+, 0)$ is a totally ordered Abelian group, and $(L ; \leqslant,+, 0)$ is a subtomonoid of $\left(G^{+} ; \leqslant,+, 0\right)$. The assertion follows.

We can characterise group cone tomonoids by means of monomial preorders as follows. Call a preorder $\preccurlyeq$ on $\mathbb{N}^{n}$ cancellative if for any $a, b, c \in \mathbb{N}^{n}, a \preccurlyeq b$ is equivalent to $a+c \preccurlyeq b+c$.

Proposition 3.3. Let the p.c. tomonoid $L$ be represented by the monomial preorder $\preccurlyeq$ on $\mathbb{N}^{n}$. Then $L$ is a group cone tomonoid if and only if $\preccurlyeq$ is cancellative.

Proof. Let $L$ be a group cone tomonoid. Then $\left(\left\langle\mathbb{N}^{n}\right\rangle_{\preccurlyeq} ; \preccurlyeq,+,\{\overline{0}\}\right)$ is cancellative by Proposition 3.2. Thus, for $a, b, c \in \mathbb{N}^{n}$, we have $a \preccurlyeq b$ iff $\langle a\rangle_{\preccurlyeq} \preccurlyeq\langle b\rangle_{\preccurlyeq}$ iff $\langle a\rangle_{\preccurlyeq}+\langle c\rangle_{\preccurlyeq} \preccurlyeq\langle b\rangle_{\preccurlyeq}+\langle c\rangle_{\preccurlyeq}$ iff $\langle a+c\rangle_{\preccurlyeq} \preccurlyeq\langle b+c\rangle_{\preccurlyeq}$ iff $a+c \preccurlyeq b+c$, that is, $\preccurlyeq$ is cancellative. 
Conversely, let $\preccurlyeq$ be cancellative. Then $\left(\left\langle\mathbb{N}^{n}\right\rangle_{\preccurlyeq} ; \preccurlyeq,+,\{\overline{0}\}\right)$ is a cancellative p.c. tomonoid and hence, by Proposition 3.2, a group cone tomonoid.

In what follows, $\left(\mathbb{Z}^{n} ;+, \overline{0}\right)$ will denote the free Abelian group generated by $n \geqslant 1$ elements. Furthermore, $\leqslant$ will be the partial order on $\mathbb{Z}^{n}$ defined according to (2.1): for $a, b \in \mathbb{Z}^{n}$, we put $a \leqslant b$ if $a+c=b$ for some $c \in \mathbb{N}^{n}$. Then $\left(\mathbb{Z}^{n} ; \sharp,+, \overline{0}\right)$ is a partially ordered group.

The order of a partially ordered Abelian group $(G ; \leqslant,+, 0)$ is uniquely determined by its positive cone $G^{+}=\{g \in G: g \geqslant 0\}$. In fact, for any $g, h \in G$, $g \leqslant h$ if and only if $h-g \in G^{+}$. We may alternatively understand the positive cone of a partially ordered group as the set of all differences of elements $g$ and $h$ such that $g \leqslant h$; indeed, $G^{+}=\{h-g: g, h \in G$ such that $g \leqslant h\}$.

Cancellative monomial preorders, which represent group cone monoids, can be described along similar lines.

Definition 3.4. Let $\preccurlyeq$ be a cancellative monomial preorder on $\mathbb{N}^{n}$. Then the set

$$
P_{\preccurlyeq}=\left\{b-a \in \mathbb{Z}^{n}: a, b \in \mathbb{N}^{n} \text { such that } a \preccurlyeq b\right\}
$$

is called the positive cone of $\preccurlyeq$.

A positive cone determines the preorder from which it is defined just as in the case of groups.

Lemma 3.5. Let $P \subseteq \mathbb{Z}^{n}$ be the positive cone of the cancellative monomial preorder $\preccurlyeq$. Then we have:

(GO) for any $a, b \in \mathbb{N}^{n}, a \preccurlyeq b$ if and only if $b-a \in P$.

Proof. By definition, $a \preccurlyeq b$ implies $b-a \in P$.

Conversely, let $b-a \in P$. Then there are $c, d \in \mathbb{N}^{n}$ such that $c \preccurlyeq d$ and $d-c=b-a$. Then $a+d=b+c \preccurlyeq b+d$, and hence $a \preccurlyeq b$.

Thus, we have for a cancellative monomial preorder $\preccurlyeq$,

$$
\begin{aligned}
P_{\preccurlyeq} & =\left\{z \in \mathbb{Z}^{n}: a \preccurlyeq b \text { for some } a, b \in \mathbb{N}^{n} \text { such that } z=b-a\right\} \\
& =\left\{z \in \mathbb{Z}^{n}: a \preccurlyeq b \text { for all } a, b \in \mathbb{N}^{n} \text { such that } z=b-a\right\} ;
\end{aligned}
$$

in fact, the first equality holds by definition and the second one by Lemma 3.5.

The positive cones of partially ordered Abelian groups are exactly the cancellative commutative monoids such that $a+b=0$ implies $a=b=0$. The positive cones of cancellative monomial preorders are characterised as follows.

Theorem 3.6. A set $P \subseteq \mathbb{Z}^{n}$ is the positive cone of a cancellative monomial preorder on $\mathbb{N}^{n}$ if and only if the following conditions are fulfilled.

(GC1) Let $z \in \mathbb{N}^{n}$. Then $z \in P$, and if $z \neq \overline{0}$, then $-z \notin P$.

(GC2) $P$ is closed under addition.

(GC3) Let $z \in \mathbb{Z}^{n}$. Then $z \in P$ or $-z \in P$.

In this case, $P=P_{\preccurlyeq}$, where $\preccurlyeq$ is given by condition (GO) above. 
Proof. Let $\preccurlyeq$ be a cancellative monomial preorder on $\mathbb{N}^{n}$. Clearly, $0 \in P_{\preccurlyeq}$ then. Furthermore, any $z \in \mathbb{N}^{n} \backslash\{\overline{0}\}$ is in $P_{\preccurlyeq}$ because $\overline{0} \preccurlyeq z$ holds by the positivity of $\preccurlyeq$. Assume that also $-z \in P_{\preccurlyeq}$. Then there is a $b \in \mathbb{N}^{n}$ such that $b+z \preccurlyeq b$ and hence by the cancellativity $z \preccurlyeq 0$, in contradiction to the positivity of $\preccurlyeq$. This shows (GC1).

For $a, b, c, d \in \mathbb{N}^{n}, a \preccurlyeq b$ and $c \preccurlyeq d$ implies $a+c \preccurlyeq b+c \preccurlyeq b+d$. We conclude that if $b-a, d-c \in P_{\preccurlyeq}$, also $(b-a)+(d-c)=(b+d)-(a+c) \in P_{\preccurlyeq}$. This shows (GC2).

For $a, b \in \mathbb{N}^{n}$, at least one of $a \preccurlyeq b$ or $b \preccurlyeq a$ holds because $\preccurlyeq$ is total. So, (GC3) follows as well.

Let now $P \subseteq \mathbb{Z}^{n}$ fulfil (GC1)-(GC3). For $a, b \in \mathbb{N}^{n}$, let $a \preccurlyeq b$ if $b-a \in P$. We claim that $\preccurlyeq$ is a cancellative monomial preorder. As $0 \in P$ by (GC1), $\preccurlyeq$ is reflexive. By $(\mathrm{GC} 2)$, $\preccurlyeq$ is transitive. Hence, $\preccurlyeq$ is a preorder. Then $\preccurlyeq$ is total by (GC3) and positive by (GC1). Finally, by construction, $a \preccurlyeq b$ is equivalent to $a+c \preccurlyeq b+c$; the compatibility and cancellativity of $\preccurlyeq$ follows.

It remains to show that $P$ is actually the positive cone $P \preccurlyeq$ of $\preccurlyeq$. By Lemma 3.5, we have that for any $a, b \in \mathbb{N}^{n}, b-a \in P_{\preccurlyeq}$ if and only if $a \preccurlyeq b$. But by construction, $a \preccurlyeq b$ if and only if $b-a \in P$. Hence, $P=P \preccurlyeq$.

Finally, if $P \subseteq \mathbb{Z}^{n}$ is the positive cone of any cancellative monomial preorder $\preccurlyeq$, then $\preccurlyeq$ is by Lemma 3.5 uniquely determined by (GO). The last statement follows.

In the context of tomonoids, we may consider a positive cone, without explicit reference to some monomial preorder, as a subset of $\mathbb{Z}^{n}$ fulfilling conditions (GC1)-(GC3). Group cone tomonoids correspond to cancellative monomial preorders; Theorem 3.6 then establishes a one-to-one correspondence between cancellative monomial preorders and positive cones.

In what follows, we will generalise the notion of a positive cone to cover a wider class of tomonoids. In this case, we will not obtain a strict correlation such as in the context of group cone tomonoids, but we will be led to a Galois correspondence.

\section{Direction cones}

In this section, we introduce a tool to describe monomial preorders. The condition of cancellativity will no longer be assumed.

Let $\preccurlyeq$ be a monomial preorder on $\mathbb{N}^{n}$ and recall for a moment again the case that $\preccurlyeq$ is cancellative. Then, for any pair $a, b \in \mathbb{N}^{n}$, the question of whether or not $a \preccurlyeq b$ holds depends only on the difference $z=b-a$ : we have $a \preccurlyeq b$ if and only if $c \preccurlyeq d$ for any other pair $c, d \in \mathbb{N}^{n}$ such that $z=d-c$. By (3.1), the positive cone $P_{\preccurlyeq}$ consists of these differences; $a \preccurlyeq b$ if and only if $b-a \in P_{\preccurlyeq}$.

In general, the question of whether or not $a \preccurlyeq b$ does not depend on their difference alone; e.g., it may be the case that $a+c \preccurlyeq b+c$ for some $c \in \mathbb{N}^{n}$, but not $a \preccurlyeq b$. However, let $z \in \mathbb{Z}^{n}$. Then we can still say that at least one 
of following possibilities applies: $a \preccurlyeq b$ for all $a, b \in \mathbb{N}^{n}$ such that $b-a=z$, or $b \preccurlyeq a$ for all $a, b \in \mathbb{N}^{n}$ such that $b-a=z$. This is a consequence of the following elementary lemma.

Lemma 4.1. Let $z \in \mathbb{Z}^{n}$. Then there is a unique pair $a, b \in \mathbb{N}^{n}$ such that $z=b-a$, and for any $c, d \in \mathbb{N}^{n}$ such that $z=d-c$, we have $c=a+t$ and $d=b+t$ for some $t \in \mathbb{N}^{n}$.

Proof. Put $a=-z \vee \overline{0}$ and $b=z \vee \overline{0}$. Then $z=b-a$. Moreover, if $c, d \in \mathbb{N}^{n}$ such that $d-c=z$, we have $c \geqslant \overline{0}$ and $c=d-z \unrhd-z$; thus, $c \geqslant a$. Similarly, $d \unrhd b$. As $b-a=d-c$, the differences $c-a$ and $d-b$ coincide. The uniqueness of $a, b$ follows from the $₫$-minimality.

With reference to Lemma 4.1 , the pair $a, b \in \mathbb{N}^{n}$ associated to an element $z \in \mathbb{Z}^{n}$ has the property that $z=b-a$, and any other pair in $\mathbb{N}^{n}$, whose difference is also $z$, arises from $a, b$ by translation by some $t \geqslant \overline{0}$. Inspecting the above proof, we see that $b$ is simply the positive part of $z \in \mathbb{Z}^{n}$, and $a$ is its (negated) negative part. We define $z^{+}=z \vee \overline{0}$ and $z^{-}=-z \vee \overline{0}$; then $z^{+}, z^{-} \in \mathbb{N}^{n}$ are such that $z=z^{+}-z^{-}$.

Let now $\preccurlyeq$ be a compatible preorder on $\mathbb{N}^{n}$ and let $z \in \mathbb{Z}^{n}$. If $z^{-} \preccurlyeq z^{+}$, we conclude from Lemma 4.1 and the compatibility of $\preccurlyeq$ that $a \preccurlyeq b$ actually holds for any pair $a, b \in \mathbb{N}^{n}$ such that $b-a=z$. Thus, intuitively, we may view any $z \in \mathbb{Z}^{n}$ such that $z^{-} \preccurlyeq z^{+}$as being "positively directed"; for, in this case, we have $a \preccurlyeq a+z$ for any $a \in \mathbb{N}^{n}$ such that $a+z \in \mathbb{N}^{n}$. Our viewpoint is reflected in the following definition.

Definition 4.2. Let $\preccurlyeq$ be a monomial preorder on $\mathbb{N}^{n}$. Then we call the set $C_{\preccurlyeq}=\left\{z \in \mathbb{Z}^{n}: z^{-} \preccurlyeq z^{+}\right\}$the direction cone of $\preccurlyeq$.

From Lemma 4.1 it is immediate that for any monomial preorder $\preccurlyeq$,

$$
C_{\preccurlyeq}=\left\{z \in \mathbb{Z}^{n}: a \preccurlyeq b \text { for all } a, b \in \mathbb{N}^{n} \text { such that } z=b-a\right\} .
$$

Comparing with (3.1), we see that the direction cone of a cancellative monomial preorder is its positive cone. In the general case, we conclude from the positivity of $\preccurlyeq$ that condition (GC1) for positive cones applies here as well, and from the totality of $\preccurlyeq$ also condition (GC3) is immediate: for each $z \in \mathbb{Z}^{n}$, at least one of $z$ or $-z$ is in $C \preccurlyeq$.

In contrast, a direction cone does not in general fulfil condition (GC2), that is, it is not necessarily closed under addition. In order to see in which respect direction cones differ from positive cones, we introduce the following notion. We call a $k$-tuple of elements of $\mathbb{Z}^{n},\left(x_{1}, \ldots, x_{k}\right)$ for $k \geqslant 2$, addable if

$$
\left(x_{1}+\cdots+x_{k}\right)^{-}+x_{1}+\cdots+x_{i} \geqslant \overline{0}
$$

for all $i=0, \ldots, k$. Note that for addability, the order matters.

The condition of addability is somewhat cumbersome, but essential for what follows. We may express it alternatively as follows. 
Lemma 4.3. A $k$-tuple $\left(x_{1}, \ldots, x_{k}\right)$ of elements of $\mathbb{Z}^{n}$, where $k \geqslant 2$, is addable if and only if for every $i=1, \ldots, k, x_{i}+\cdots+x_{k} \sharp\left(x_{1}+\cdots+x_{k}\right) \vee \overline{0}$.

Proof. Note first that condition (4.2) always holds if $i=k$; for, the left side equals $\left(x_{1}+\cdots+x_{k}\right)^{+}$in this case.

Putting $z=x_{1}+\cdots+x_{k},\left(x_{1}, \ldots, x_{k}\right)$ is hence addable if and only if $z^{-}+x_{1}+\cdots+x_{i} \triangleq \overline{0}$ for all $i=0, \ldots, k-1$. This is in turn is equivalent to saying that $z^{-}+x_{1}+\cdots+x_{k} \geqslant x_{i+1}+\cdots+x_{k}$ for all $i=0, \ldots, k-1$. But $z^{-}+x_{1}+\cdots+x_{k}=z^{-}+z=z^{+}=z \vee \overline{0}$, and the assertion follows.

The next lemma contains the characteristic properties of direction cones.

Lemma 4.4. The direction cone of a monomial preorder on $\mathbb{N}^{n}$ is a set $C \subseteq$ $\mathbb{Z}^{n}$ fulfilling the following conditions.

(C1) Let $z \in \mathbb{N}^{n}$. Then $z \in C$, and if $z \neq \overline{0}$, then $-z \notin C$.

(C2) Let $\left(x_{1}, \ldots, x_{k}\right)$ for $k \geqslant 2$ be an addable $k$-tuple of elements of $C$. Then $x_{1}+\cdots+x_{k} \in C$.

(C3) Let $z \in \mathbb{Z}^{n}$. Then $z \in C$ or $-z \in C$.

Proof. (C1): We have $\mathbb{N}^{n} \subseteq C$ because $\preccurlyeq$ is positive. Assume that $-z \in C$, where $z \in \mathbb{N}^{n}$. Then $z=(-z)^{-} \preccurlyeq(-z)^{+}=\overline{0}$ and the positivity of $\preccurlyeq$ implies $z=\overline{0}$.

Recall next that by (4.1), $a \preccurlyeq b$ for any $a, b \in \mathbb{N}^{n}$ such that $b-a \in C$.

(C2): Let $\left(x_{1}, \ldots, x_{k}\right)$ be as indicated, and put $z=x_{1}+\cdots+x_{k}$. Then $z^{-}, z^{-}+x_{1}, \ldots, z^{-}+x_{1}+\cdots+x_{k} \in \mathbb{N}^{n}$. By assumption, $x_{1}, \ldots, x_{k} \in C$; thus, $z^{-} \preccurlyeq z^{-}+x_{1} \preccurlyeq \cdots \preccurlyeq z^{-}+x_{1}+\cdots+x_{k}=z^{-}+z=z^{+}$.

(C3): This holds because $\preccurlyeq$ is total.

A further property of direction cones is the following.

Lemma 4.5. Let $\preccurlyeq$ be a monomial preorder on $\mathbb{N}^{n}$. Then $z \in C_{\preccurlyeq}$ and $a \unrhd \overline{0}$ imply $z+a \in C_{\preccurlyeq}$.

Proof. Let $z \in C_{\preccurlyeq}$ and $a \unrhd \overline{0}$. Then

$(z+a)^{-}=(-z-a) \vee \overline{0} \preccurlyeq-z \vee \overline{0}=z^{-} \preccurlyeq z^{+}=z \vee \overline{0} \preccurlyeq(z+a) \vee \overline{0}=(z+a)^{+} ;$ hence, $z+a \in C_{\preccurlyeq}$.

A preorder gives rise to a direction cone; conversely, we can assign a preorder to a set fulfilling $(\mathrm{C} 1)-(\mathrm{C} 3)$.

Definition 4.6. Let $C \subseteq \mathbb{Z}^{n}$ fulfil $(\mathrm{C} 1)-(\mathrm{C} 3)$. Let $\preccurlyeq C$ be the smallest preorder on $\mathbb{N}^{n}$ such that

(O) $a \preccurlyeq_{C} b$ for any $a, b \in \mathbb{N}^{n}$ such that $b-a \in C$.

Then we call $\preccurlyeq_{C}$ the monomial preorder induced by $C$.

More explicitly, given $C \subseteq \mathbb{Z}^{n}$ and $a, b \in \mathbb{N}^{n}$, we have $a \preccurlyeq_{C} b$ if and only if there are $k \geqslant 1$ elements $z_{1}, \ldots, z_{k} \in C$ such that $a, a+z_{1}, a+z_{1}+z_{2}, \ldots$, $a+z_{1}+\cdots+z_{k} \geqslant \overline{0}$ and $a+z_{1}+\cdots+z_{k}=b$. Note that if $b-a$ is the sum of elements of $C$, it is not automatic that $a \preccurlyeq b$. 
Lemma 4.7. Let $C \subseteq \mathbb{Z}^{n}$ fulfil $(\mathrm{C} 1)-(\mathrm{C} 3)$. Then $\preccurlyeq C$, the monomial preorder induced by $C$, is in fact a monomial preorder.

Proof. By construction, $\preccurlyeq_{C}$ is a preorder, and by (C3), $\preccurlyeq_{C}$ is total. It is furthermore clear that $\preccurlyeq C$ is compatible with the addition.

Assume next that for some $a \in \mathbb{N}^{n}, a \preccurlyeq_{C} \overline{0}$ holds according to the prescription (O). Then $a=\overline{0}$ by (C1). It follows that $\overline{0} \prec_{C} a$ for all $a \in \mathbb{N}^{n} \backslash\{\overline{0}\}$, that is, $\preccurlyeq_{C}$ is positive. This completes the proof that $\preccurlyeq_{C}$ is a monomial preorder.

We now show that the properties (C1)-(C3) of Lemma 4.4 exactly characterise monomial preorders.

Theorem 4.8. A set $C \subseteq \mathbb{Z}^{n}$ is the direction cone of a monomial preorder if and only if $C$ fulfils $(\mathrm{C} 1)-(\mathrm{C} 3)$. In this case, $C$ is the direction cone of $\preccurlyeq C$.

Proof. A direction cone fulfils (C1)-(C3) by Lemma 4.4.

Conversely, let $C$ fulfil $(\mathrm{C} 1)-(\mathrm{C} 3)$. Let $\preccurlyeq_{C}$ be the induced preorder. By Lemma $4.7, \preccurlyeq_{C}$ is a monomial preorder.

It remains to show that $C_{\preccurlyeq_{C}}$, the direction cone of $\preccurlyeq_{C}$, coincides with $C$, that is, for $z \in \mathbb{Z}^{n}, z^{-} \preccurlyeq C z^{+}$if and only if $z \in C$. The "if" part holds by construction. For the "only if" part, assume that $z^{-} \preccurlyeq C z^{+}=z^{-}+z$. Then $z=x_{1}+\cdots+x_{k}$ for some $x_{1}, \ldots, x_{k} \in C$ such that $z^{-}+x_{1}+\cdots+x_{i} \geqslant \overline{0}$ for $i=0, \ldots, k$. Then $\left(x_{1}, \ldots, x_{k}\right)$ is addable, hence $z \in C$ by $(\mathrm{C} 2)$.

In the sequel, when speaking about direction cones without reference to a monomial preorder, we mean a subset of $\mathbb{Z}^{n}$ that fulfils conditions (C1)-(C3).

A direction cone induces a preorder. As seen next, any preorder contains a preorder arising in this way.

Theorem 4.9. Let $\preccurlyeq$ be a monomial preorder. Then $\preccurlyeq$ extends $\preccurlyeq C_{\preccurlyeq}$, the monomial preorder induced by the direction cone of $\preccurlyeq$.

Moreover, the direction cone of $\preccurlyeq C_{\preccurlyeq}$ is $C_{\preccurlyeq}$ again.

Proof. Let $a, b \in \mathbb{N}^{n}$ and assume that $a \preccurlyeq C_{\preccurlyeq} b$ holds according to the prescription (O). Then $b-a \in C_{\preccurlyeq}$, that is, $z^{-} \preccurlyeq z^{+}$, where $z=b-a$. In view of Lemma 4.1, it follows $a \preccurlyeq b$. We conclude that $\preccurlyeq C_{\preccurlyeq} \subseteq \preccurlyeq$.

The second part holds by Theorem 4.8 .

A Galois connection between monomial preorders and direction cones. For a given $n \geqslant 1$, let $\mathcal{P}$ be the set of all monomial preorders on $\mathbb{N}^{n}$ and let $\mathcal{C}$ be the set of all direction cones in $\mathbb{Z}^{n}$. Note that the two mappings

$$
\mathcal{P} \rightarrow \mathcal{C}, \quad \preccurlyeq \mapsto C_{\preccurlyeq}, \quad \mathcal{C} \rightarrow \mathcal{P}, \quad C \mapsto \preccurlyeq C
$$

are order-preserving, with respect to set-theoretic inclusion. The mappings are not one-to-one; in fact, the former is surjective but not injective, and the latter is injective but not surjective. But Theorems 4.8 and 4.9 indicate the result of applying the mappings successively: any $\preccurlyeq \in \mathcal{P}$ is an extension of 
$\preccurlyeq C_{\preccurlyeq}$; and any $C \in \mathcal{C}$ is equal to $C_{\preccurlyeq}$. We conclude that there is a Galois connection between $\mathcal{P}$ and $\mathcal{C}$. Namely, for any $\preccurlyeq \in \mathcal{P}$ and $C \in \mathcal{C}$,

$$
\preccurlyeq C \subseteq \preccurlyeq \quad \text { if and only if } C \subseteq C_{\preccurlyeq} .
$$

We apply the shown facts to tomonoids.

Definition 4.10. Let $C \subseteq \mathbb{Z}^{n}$ be a direction cone. Then we call the tomonoid represented by $\preccurlyeq C$ a cone tomonoid.

Theorem 4.11. Each finitely generated p.c. tomonoid $L$ is the quotient of a cone tomonoid.

Proof. This holds by Theorem 4.9 and Lemma 2.8 .

Example. Let us present an example illustrating the results of this section. Let $L$ be the 9 -element p.c. tomonoid specified as follows. Let $L$ be generated by its two elements $a$ and $b$; assume that

$$
\begin{aligned}
0<a<b & <2 a<a+b<2 b<3 a \\
& <2 a+b=a+2 b=4 a<2 a+2 b=3 a+b=5 a=3 b
\end{aligned}
$$

and that the last indicated element is the top element. In accordance with Proposition 2.7, let $\iota: \mathbb{N}^{2} \rightarrow L$ be the surjective monoid homomorphism such that $\iota((1,0))=a$ and $\iota((0,1))=b$, and endow $\mathbb{N}^{2}$ with the preorder $\preccurlyeq$ according to (2.3). Then we have

$$
\begin{aligned}
(0,0) \prec(1,0) \prec(0,1) & \prec(2,0) \prec(1,1) \prec(0,2) \\
& \prec(3,0) \prec(2,1) \approx(1,2) \approx(4,0) \prec(m, n),
\end{aligned}
$$

where $(m, n)$ is any of the remaining elements of $\mathbb{N}^{2}$. A graphical representation of $(L ; \leqslant,+, 0)$ can be found in Figure 1 .

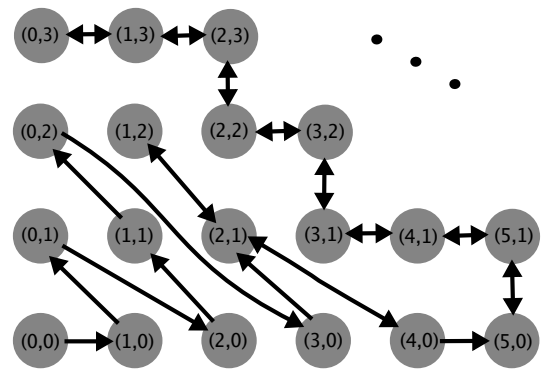

Figure 1 . The example tomonoid $L$. The simple arrows indicate the immediate-successor relation with respect to $\preccurlyeq$; the double arrows indicate $\preccurlyeq$-equivalence.

The direction cone is, by Definition 4.2 ,

$$
C_{\preccurlyeq}=\left\{(p, q) \in \mathbb{Z}^{2}:(-p \vee 0,-q \vee 0) \preccurlyeq(p \vee 0, q \vee 0)\right\} .
$$


Thus, for $m, n \in \mathbb{N}$, we have $(m, n) \in C_{\preccurlyeq} ;(-m, n) \in C_{\preccurlyeq}$ if $(m, 0) \preccurlyeq(0, n)$; and $(m,-n) \in C_{\preccurlyeq}$ if $(0, n) \preccurlyeq(m, 0)$. We get

$$
\begin{aligned}
C_{\preccurlyeq}= & \left\{(p, q) \in \mathbb{Z}^{2}: p, q \geqslant 0\right\} \\
& \cup\{(-2,2),(-1,1),(-1,2),(2,-1),(3,-2),(3,-1),(4,-2),(4,-1)\} \\
& \cup\left\{(p, q) \in \mathbb{Z}^{2}: p \leqslant 0 \text { and } q \geqslant 3\right\} \cup\left\{(p, q) \in \mathbb{Z}^{2}: p \geqslant 5 \text { and } q \leqslant 0\right\},
\end{aligned}
$$

depicted in Figure 2.

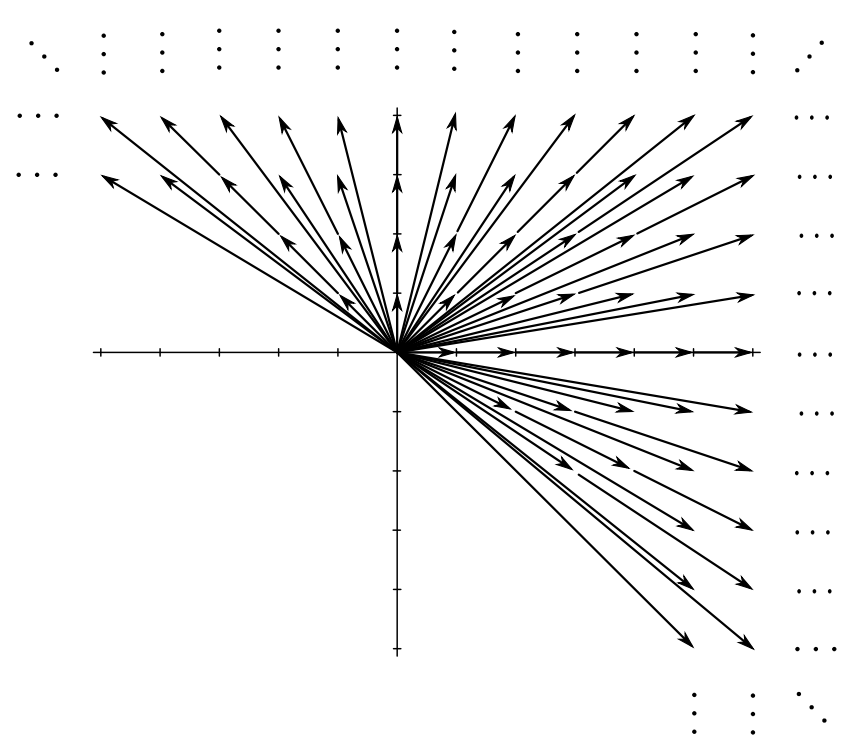

Figure 2. The direction cone $C_{\preccurlyeq}$ of the monomial preorder $\preccurlyeq$ representing $L$. Each element of $C \preccurlyeq$ is depicted as a vector based at $(0,0)$.

Finally, we calculate $\preccurlyeq C_{\preccurlyeq}$, the preorder representing a cone tomonoid whose quotient is $L$. The preorder $\preccurlyeq C_{\preccurlyeq}$ can most easily be read off directly from Figure 1. Namely, we collect the order relations that hold between elements of the form $(m, 0)$ and $(0, n)$, where $m, n \geqslant 1$; then we translate and concatenate them. The result is depicted in Figure 3 . From $\preccurlyeq_{C_{\preccurlyeq}}$, we get $L$ by requiring the elements $(2,1),(1,2)$, and $(4,0)$ of $\mathbb{N}^{2}$ to be equivalent.

\section{Formally integral tomonoids}

In this section, we briefly reconsider group cone tomonoids and we then see what in the present context the condition of formal integrality means.

Proposition 5.1. A group cone tomonoid is a cone tomonoid.

Proof. The direction cone of a monomial preorder $\preccurlyeq$ representing a group cone tomonoid is its positive cone $P_{\preccurlyeq}$, and $P_{\preccurlyeq}$ induces $\preccurlyeq$. 


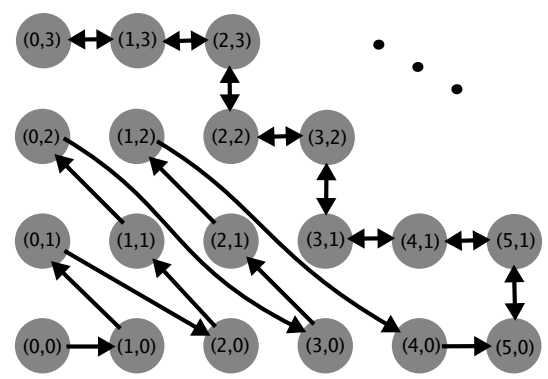

Figure 3 . The cone tomonoid represented by $\preccurlyeq C_{\preccurlyeq}$, whose quotient is $L$.

Proposition 5.2. Let $C \subseteq \mathbb{Z}^{n}$ be a direction cone. Then the cone tomonoid represented by $\preccurlyeq_{C}$ is a group cone tomonoid if and only if $C$ is closed under addition if and only if $\preccurlyeq_{C}$ is cancellative.

Proof. By Proposition $3.3, \preccurlyeq_{C}$ represents a group cone tomonoid if and only if $\preccurlyeq C$ is cancellative.

Assume that $C$ is closed under addition. Then, for $a, b \in \mathbb{N}^{n}, a \preccurlyeq_{C} b$ is equivalent to $b-a \in C$, and it follows that $\preccurlyeq_{C}$ is cancellative.

Furthermore, assume that $\preccurlyeq C$ is cancellative. To see that $C$ is closed under addition, let $x, y \in C$. Then $x^{-} \preccurlyeq_{C} x^{+}=x+x^{-}$and $y^{-} \preccurlyeq_{C} y+y^{-}$, and hence $x^{-}+y^{-} \preccurlyeq C x^{-}+y^{-}+x+y$. From $(x+y)^{-} \preccurlyeq x^{-}+y^{-}$and the cancellativity of $\preccurlyeq C$, we conclude $(x+y)^{-} \preccurlyeq_{C}(x+y)^{-}+x+y$; hence, $x+y \in C$.

We now turn to the topic of formal integrality. For a detailed discussion of this property, we refer to [4]; see also [9].

Definition 5.3. Let $(L ; \leqslant,+, 0)$ be a p.c. tomonoid. If there is a total order $\leqslant$ on $\mathbb{N}^{n}$ such that $\left(\mathbb{N}^{n} ; \leqslant,+, 0\right)$ is a p.c. tomonoid and $L$ is isomorphic to a tomonoid quotient of $\mathbb{N}^{n}$, then $L$ is called formally integral.

We note that formal integrality is usually defined in a more general context, namely, without the assumption of positivity. It is not difficult to check that our definition is consistent with the definition given, e.g., in [4].

If we want to avoid the explicit reference to some $\mathbb{N}^{n}$, we may characterise formal integrality also as follows.

Proposition 5.4. Let $(L ; \leqslant,+, 0)$ be a finitely generated p.c. tomonoid. Then $L$ is formally integral if and only if $L$ is the quotient of a group cone tomonoid.

Proof. Let $\left(\mathbb{N}^{n} ; \leqslant,+, \overline{0}\right)$ be a p.c. tomonoid. Then $\mathbb{N}^{n}$ is a group cone tomonoid because $\leqslant$ is cancellative. The "only if" part follows.

Conversely, let $L$ be a group cone tomonoid. Then $L$ is cancellative, and hence by $[4$, Cor. 4.5], formally integral. The "if" part follows as well.

We will call a monomial preorder that is actually a partial order a monomial order, in accordance with the common meaning of this notion [2]. Furthermore, 
we will call a subset $P \subseteq \mathbb{Z}^{n}$ a $\mathbb{Z}^{n}$ cone if $P$ fulfils (GC1), (GC2), and the following strengthening of (GC3): for each $z \in \mathbb{Z}^{n}$, exactly one of $z$ and $-z$ is in $P$.

Theorem 5.5. Let $L$ be a finitely generated p.c. tomonoid. Then the following conditions are pairwise equivalent.

(i) $L$ is formally integral.

(ii) $L$ is represented by a monomial preorder that contains a monomial order.

(iii) $L$ is represented by a monomial preorder $\preccurlyeq$ such that $C_{\preccurlyeq}$ contains a $\mathbb{Z}^{n}$ cone.

Proof. (i) $\Longrightarrow$ (ii): Let $L$ be a quotient of the p.c. tomonoid $\left(\mathbb{N}^{n} ; \leqslant,+, \overline{0}\right)$. Without loss of generality, we can assume that the quotient is pure. Moreover, $\leqslant$ is a monomial order. By Lemma 2.8, there is a monomial preorder $\preccurlyeq \supseteq \leqslant$ on $\mathbb{N}^{n}$ that represents $L$; (ii) follows.

(ii) $\Longrightarrow$ (iii): Let $\preccurlyeq$ be a monomial preorder on $\mathbb{N}^{n}$ representing $L$, and let $\leqslant \subseteq \preccurlyeq$ be a monomial order. Then $\left(\mathbb{N}^{n} ; \leqslant,+, \overline{0}\right)$ is a p.c. tomonoid whose direction cone is $C_{\leqslant}=\left\{z \in \mathbb{Z}^{n}: z^{-} \leqslant z^{+}\right\} \subseteq C_{\preccurlyeq}$. Since $\left(\mathbb{N}^{n} ; \leqslant,+, \overline{0}\right)$ is a group cone tomonoid, $C_{\leqslant}$fulfils (GC1)-(GC3). Moreover, for any $z \in \mathbb{Z}^{n} \backslash\{\overline{0}\}$, $z \in C_{\leqslant}$contradicts $-z \in C_{\leqslant}$. Hence, $C_{\leqslant}$is a $\mathbb{Z}^{n}$ cone; (iii) follows.

(iii) $\Longrightarrow$ (i): Let $\preccurlyeq$ be a monomial preorder on $\mathbb{N}^{n}$ representing $L$, and let $C \subseteq C_{\preccurlyeq}$ be a $\mathbb{Z}^{n}$ cone. Then the monomial preorder induced by $C$ is in fact a monomial order $\leqslant$, and $\left(\mathbb{N}^{n} ; \leqslant,+, \overline{0}\right)$ is a p.c. tomonoid. Furthermore, $\leqslant \subseteq \preccurlyeq$; hence, by Lemma $2.8, L$ is a tomonoid quotient of $\mathbb{N}^{n}$, and (i) is proved.

Let us briefly summarise what we have established so far. Cone tomonoids are those p.c. tomonoids that are represented by direction cones. Group cone tomonoids are the cancellative cone tomonoids and they are represented by positive cones, which are those direction cones that are closed under addition. Furthermore, the quotients of cone tomonoids comprise all p.c. tomonoids. The quotients of group cone tomonoids are the formally integral p.c. tomonoids.

Example. Theorem 5.5 provides an easily verifiable sufficient criterion that a tomonoid is not formally integral. Assume that some direction cone $C$ contains the elements $x$ and $y$, but neither $-x$ nor $-y$ nor $x+y$. If $C$ represented a formally integral p.c. tomonoid, it would contain a $\mathbb{Z}^{n}$ cone; but this is impossible as the latter would contain $x$ and $y$ and thus also $x+y$.

As an example, consider the well-known 9-element non-formally integral tomonoid presented in [4]. Let $L$ be generated by the three elements $a, b$, and $c$ such that

$$
0<a<b<c<2 a<a+b=2 b<3 a=a+c<b+c=2 c<4 a,
$$

and all non-indicated sums are equal to the top element, which is $4 a$. 


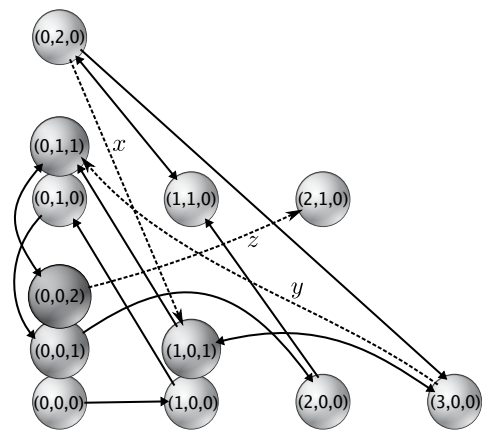

Figure 4. The 9-element example tomonoid from [4].

To represent $L$ by a preorder in $\mathbb{N}^{3}$, we assign $(1,0,0)$ to $a,(0,1,0)$ to $b$, and $(0,0,1)$ to $c$. Then the direction cone $C$ of the representing preorder contains

$$
\begin{array}{ll}
x=(1,-2,1) & \text { because } 2 b<a+c, \\
y=(-3,1,1) & \text { because } 3 a<b+c, \\
z=(2,1,-2) & \text { because } 2 c<2 a+b,
\end{array}
$$

and $C$ does not contain any of $-x,-y,-z$ because the inequalities are strict. Thus, $C$ contains $x$ and $y$ but neither $-x$ nor $-y$ nor $-z=x+y$. An illustration of $L$ can be found in Figure 4 .

Note furthermore that the absence of $x+y$ in $C$ implies that neither $(x, y)$ nor $(y, x)$ are addable. In fact, starting from $(x+y)^{-}=(2,1,0)$ and adding $x$ and then $y$, or $y$ and then $x$, we arrive at $(x+y)^{+}=(0,0,2)$, and in both cases, we pass through an element outside $\mathbb{N}^{3}$.

\section{Nilpotent finite tomonoids}

By Theorem 4.11, any finitely generated p.c. tomonoid is the quotient of a cone tomonoid. Cone tomonoids are in turn completely described by their direction cones, which are subsets of $\mathbb{Z}^{n}$ characterised by Theorem 4.8 .

Finite p.c. tomonoids are, in particular, included in our discussion. However, the direction cone associated with a finite p.c. tomonoid is in general not finite. We shall see in the present section that this drawback can be overcome quite easily if the tomonoid in question is nilpotent.

Definition 6.1. A p.c. tomonoid $(L ; \leqslant,+, 0)$ is called nilpotent if $L$ possesses a greatest element $\tau$ and there is a $k \geqslant 1$ such that any sum of at least $k$ elements distinct from 0 equals $\tau$.

We note that nilpotency is a notion applicable to monoids in general; see, e.g., [7]. A monoid $(L ;+, 0)$ is called nilpotent if for some $k \geqslant 1$, the sums of at least $k$ non-zero elements are all equal. We further note that, in our context, nilpotency is closely related to the Archimedean property. In contrast 
to nilpotency, Archimedeanicity is an order-theoretic notion; see, e.g., [5]. A p.c. tomonoid $(L ; \leqslant,+, 0)$ is called Archimedean if for any $a, b \in L \backslash\{0\}, a \leqslant b$ implies that $b \leqslant k a$ for some $k \geqslant 1$. Here, we shall discuss finitely generated nilpotent p.c. tomonoids. Such tomonoids are finite, and a finite p.c. tomonoid is obviously nilpotent if and only if it is Archimedean.

Nilpotent finite commutative monoids have been studied by several authors and several ways for their description have been proposed. Our own procedure is inspired by P. A. Grillet's method, which is explained in [7, Chapter IX].

The starting point is the following. Congruences on $\mathbb{N}^{n}$ inducing a nilpotent monoid have exactly one infinite class, namely the class of the top element, whereas all other classes are finite. To describe a nilpotent tomonoid, it is therefore sufficient to consider the finite congruence classes only, and this is what we will do here.

A monomial preorder representing a nilpotent tomonoid will be called nilpotent as well. Our topic is, accordingly, the nilpotent monomial preorders.

In what follows, a $₫$-ideal will be a non-empty subset $A$ of $\mathbb{N}^{n}$ such that $a \in A$ and $b \leqslant a$ imply $b \in A$. Furthermore, $A \subseteq \mathbb{N}^{n}$ is said to be closed under translations by some $z \in \mathbb{Z}^{n}$ if $a \in A$ and $a+z \in \mathbb{N}^{n}$ always imply $a+z \in A$.

Finally, given a monomial preorder $\preccurlyeq$ representing a finite tomonoid, we will, for simplicity, denote the top congruence class by $\langle\tau\rangle_{\preccurlyeq}$.

Definition 6.2. Let $\preccurlyeq$ be a nilpotent monomial preorder on $\mathbb{N}^{n}$. Then we call $S_{\preccurlyeq}=\left\{a \in \mathbb{N}^{n}: a \prec a+u\right.$ for some $\left.u \in U\left(\mathbb{N}^{n}\right)\right\}$ the support of $\preccurlyeq$.

In what follows, we denote the set-theoretic complement in $\mathbb{N}^{n}$ by the symbol $\complement$.

Lemma 6.3. Let $\preccurlyeq$ be a nilpotent monomial preorder on $\mathbb{N}^{n}$; the following hold.

(i) Each finite $\preccurlyeq$-class is a subset of $S_{\preccurlyeq}$ consisting of pairwise $₫$-incomparable elements.

(ii) The union of the finite $\preccurlyeq$-classes is $S_{\preccurlyeq}$, and $S_{\preccurlyeq}$ is a finite $\checkmark$-ideal in $\mathbb{N}^{n}$.

(iii) The top congruence class $\langle\tau\rangle_{\preccurlyeq}$ is the only infinite class and equals $\complement S_{\preccurlyeq}$. Moreover, there are pairwise $₫$-incomparable elements $a_{1}, \ldots, a_{k} \in \mathbb{N}^{n}$ such that $\langle\tau\rangle_{\preccurlyeq}=\bigcup_{i=1}^{k}\left(a_{i}+\mathbb{N}^{n}\right)$.

Proof. Let $a \notin S_{\preccurlyeq}$. Then $a \approx a+u$ for any $u \in U\left(\mathbb{N}^{n}\right)$, and consequently $a \approx a+u \approx a+2 u \approx \cdots$. By nilpotency, there is a $k \geqslant 1$ such that the sum of at least $k$ non-zero elements of the tomonoid represented by $\preccurlyeq$ is the top element. It follows that $a$ is contained in the top equivalence class $\langle\tau\rangle_{\preccurlyeq}$.

Conversely, let $a \in\langle\tau\rangle_{\preccurlyeq}$. Then $\langle a\rangle_{\preccurlyeq}=\langle a\rangle_{\preccurlyeq}+\langle u\rangle_{\preccurlyeq}=\langle a+u\rangle_{\preccurlyeq}$, that is, $a \approx a+u$ for any $u \in U\left(\mathbb{N}^{n}\right)$. It follows that $a \notin S_{\preccurlyeq}$.

We have shown that $\complement S_{\preccurlyeq}=\langle\tau\rangle_{\preccurlyeq}$. As $a \in\langle\tau\rangle_{\preccurlyeq}$ and $a \preccurlyeq b$ implies $b \in\langle\tau\rangle_{\preccurlyeq}$, it also follows that $S_{\preccurlyeq}$ is a $\Vdash$-ideal. Moreover, as the sums of at least $k$ elements of $U\left(\mathbb{N}^{n}\right)$ is in $\langle\tau\rangle_{\preccurlyeq}, S_{\preccurlyeq}$ is finite. In particular, $S_{\preccurlyeq}$ is the union of the finite $\preccurlyeq$-classes. 
We have shown (ii) and the first parts of (i) and (iii). If $a \approx a+b$ for some $a, b \in \mathbb{N}^{n}$, we have $a \approx a+b \approx a+2 b \approx \cdots$, and hence $a \in\langle\tau\rangle_{\preccurlyeq}$. The second part of (i) follows as well.

Finally, $\langle\tau\rangle_{\preccurlyeq}=\complement S_{\preccurlyeq}$ contains finitely many $\preccurlyeq$-minimal elements; the second part of (iii) follows as well.

We summarise that a nilpotent monomial preorder has finitely many finite equivalence classes, whose union is its support. Furthermore, it has exactly one infinite equivalence class, which is the complement of its support.

We note the last part of Lemma 6.3 can also be seen from a well-known result of Eilenberg and Schützenberger on congruences on free commutative monoids [3]. Namely, each $\sim$-class of a congruence $\sim$ on $\mathbb{N}^{n}$ is uniformly semilinear, that is, of the form $\bigcup_{i=1}^{k}\left(a_{i}+B^{\star}\right)$, where $a_{1}, \ldots, a_{k} \in \mathbb{N}^{n}$ and $B^{\star}$ is the submonoid generated by a finite subset $B$ of $\mathbb{N}^{n}$.

We next characterise the subsets of $\mathbb{N}^{n}$ that occur as the supports of nilpotent monomial preorders.

Theorem 6.4. A set $S \subseteq \mathbb{N}^{n}$ is the support of a nilpotent monomial preorder if and only if $S$ is a finite $₫$-ideal fulfilling the following condition:

(S) Let $z \in \mathbb{Z}^{n}$. Then $S$ is closed under translations by $z$ or $S$ is closed under translations by $-z$.

Proof. Let $S_{\preccurlyeq}$ be the support of the nilpotent monomial preorder $\preccurlyeq$. By Lemma $6.3\left(\right.$ ii),$S_{\preccurlyeq}$ is a finite $₫$-ideal. Moreover, let $z \in \mathbb{Z}^{n} \backslash\{\overline{0}\}$, and let $S_{\preccurlyeq}$ be not closed under translations by $z$. Then there is an $a \in S_{\preccurlyeq}$ such that $a+z \in \complement S_{\preccurlyeq}$, and it follows that $a \prec a+z$; we conclude that $z \in C_{\preccurlyeq}$ and $-z \notin C_{\preccurlyeq}$. If $S_{\preccurlyeq}$ is not closed under translations by $-z$ either, we conclude the opposite statement. So, (S) follows.

For the converse direction, assume that $S$ is a finite $₫$-ideal fulfilling (S). Let $\preccurlyeq$ be the smallest preorder such that the following holds: (1) for $a, b \in S$, let $a \preccurlyeq b$ if $\complement S$ is closed under translation by $b-a$; (2) for $a \in \mathbb{N}^{n}$ and $b \in \complement S$, let $a \preccurlyeq b$.

For any $z \in \mathbb{Z}^{n}, S$ is closed under translations by $z$ if and only if $C S$ is closed under translations by $-z$. Hence, $(\mathrm{S})$ implies that $\preccurlyeq$ is a total preorder.

To see that $\preccurlyeq$ is compatible with the addition, let $a, b, c \in \mathbb{N}^{n}$. Assume first that $a \preccurlyeq b$ holds according to (1), that is, $a, b \in S$ and $\complement S$ is closed under translation by $b-a$. If then $a+c \notin S$, also $b+c \notin S$ because $b+c=a+c+(b-a)$; hence, $a+c \preccurlyeq b+c$ by (2). If $b+c \notin S$, again $a+c \preccurlyeq b+c$ by (2). If finally $a+c, b+c \in S$, we have $a+c \preccurlyeq b+c$ by (1) because $(b+c)-(a+c)=b-a$. Assume second that $a \preccurlyeq b$ holds according to (2), that is, $b \notin S$. Then $b+c \notin S$ because $S$ is a $\preccurlyeq$-ideal; hence, $a+c \preccurlyeq b+c$ by (2). We conclude that $\preccurlyeq$ is a compatible preorder.

Finally, $S$ is not closed under translations by any $a \in \mathbb{N}^{n} \backslash\{\overline{0}\}$; hence, $\complement S$ is not closed under translations by $-a$ for any $a \in \mathbb{N}^{n} \backslash\{\overline{0}\}$. Moreover, $\overline{0} \notin \complement S$. 
We conclude that $a \preccurlyeq \overline{0}$ implies $a=\overline{0}$, and it follows that $\preccurlyeq$ is a positive preorder. The proof is complete that $\preccurlyeq$ is a monomial preorder.

Finally, by construction, $\complement S$ is a $\preccurlyeq$-class, and $S$ is finite. We conclude that $\preccurlyeq$ represents a nilpotent tomonoid, that is, $\preccurlyeq$ is nilpotent.

We now adapt the definition of a direction cone to the present context. Let us call $\mathcal{D}(S)=\left\{z \in \mathbb{Z}^{n}: z^{-}, z^{+} \in S\right\}$ the difference set of a $₫$-ideal $S \subseteq \mathbb{N}^{n}$.

Definition 6.5. Let $\preccurlyeq$ be a nilpotent monomial preorder on $\mathbb{N}^{n}$. Then the direction $f$-cone of $\preccurlyeq$ is the pair $\left(S_{\preccurlyeq}, F_{\preccurlyeq}\right)$, where $S_{\preccurlyeq} \subseteq \mathbb{N}^{n}$ is the support of $\preccurlyeq$ and $F_{\preccurlyeq}=\left\{z \in \mathcal{D}\left(S_{\preccurlyeq}\right): z^{-} \preccurlyeq z^{+}\right\}$.

Here, the "f" stands for "finite".

Lemma 6.6. Let $(S, F)=\left(S_{\preccurlyeq}, F_{\preccurlyeq}\right)$ be the direction f-cone of a nilpotent monomial preorder $\preccurlyeq$ on $\mathbb{N}^{n}$. Then $F$ is a subset of $\mathcal{D}(S)$ fulfilling the following conditions.

(Cf1) Let $z \in \mathcal{D}(S) \cap \mathbb{N}^{n}$. Then $z \in F$ and if $z \neq \overline{0}$, then $-z \notin F$.

(Cf2) Let $\left(x_{1}, \ldots, x_{k}\right)$, for $k \geqslant 2$, be an addable $k$-tuple of elements of $F$ whose sum is in $\mathcal{D}(S)$. Then $x_{1}+\cdots+x_{k} \in F$.

(Cf3) Let $z \in \mathcal{D}(S)$. Then $z \in F$ or $-z \in F$.

(Cf4) Let $z \in F$. Then $S$ is closed under translations by $-z$.

Proof. (Cf1)-(Cf3): These are proved similarly to the analogous statements of Lemma 4.4.

(Cf4): This follows from the fact that $S=\complement\langle\tau\rangle_{\preccurlyeq}$.

Direction cones give rise to monomial preorders; analogously, we now define preorders associated to direction f-cones.

Definition 6.7. Let $S \subseteq \mathbb{N}^{n}$ be a finite $\triangleleft$-ideal fulfilling (S), and let $F \subseteq \mathcal{D}(S)$ fulfil (Cf1)-(Cf4). Let $\preccurlyeq S, F$ be the smallest preorder on $\mathbb{N}^{n}$ such that

(Of1) $a \preccurlyeq S, F b$ for any $a, b \in S$ such that $b-a \in F$, and

(Of2) $a \preccurlyeq s, F b$ whenever $b \notin S$.

Then we call $\preccurlyeq S, F$ the monomial preorder induced by $(S, F)$.

Lemma 6.8. Let $S \subseteq \mathbb{N}^{n}$ be a finite $\sharp$-ideal fulfilling ( $\left.\mathrm{S}\right)$, and let $F \subseteq \mathcal{D}(S)$ fulfil (Cf1)-(Cf4). Then $\preccurlyeq S, F$ is a nilpotent monomial preorder.

Proof. By construction, $\preccurlyeq S, F$ is a preorder. By (Cf3), $\preccurlyeq S, F$ is total. We proceed analogously to the proof of Theorem 6.4 to see that $\preccurlyeq S, F$ is compatible with the addition. Finally, it follows from (Cf1) that $\preccurlyeq S, F$ is positive. That is, $\preccurlyeq S, F$ is a monomial preorder.

Finally, $\complement S$ is cofinite and by (Of2), consists of pairwise $\preccurlyeq S, F$-equivalent elements. Hence, $\preccurlyeq S, F$ is nilpotent.

We can now characterise the direction f-cones of nilpotent monomial preorders. 
Theorem 6.9. $(S, F) \subseteq \mathbb{N}^{n} \times \mathbb{Z}^{n}$ is the direction $f$-cone of a nilpotent monomial preorder if and only if $S$ is a finite $₫$-ideal of $\mathbb{N}^{n}$ fulfilling (S) and $F$ is a subset of $\mathcal{D}(S)$ fulfilling $(\mathrm{Cf} 1)-(\mathrm{Cf} 4)$. In this case, $(S, F)$ is the direction f-cone of $\preccurlyeq S, F$.

Proof. The "only if" part follows from Theorem 6.4 and Lemma 6.6.

To see the "if" part, let $(S, F)$ be such that the indicated conditions hold. By Lemma $6.8, \preccurlyeq S, F$ is then a nilpotent monomial order. We have to show that $(S, F)$ is its direction $\mathrm{f}$-cone.

By (Of2), $a \approx_{S, F} b$ for all $a, b \in \complement S$. Moreover, for $a \in S$ and $b \in \complement S$, we have $a \preccurlyeq S, F \quad b$; by (Cf4), $C S$ is closed under translation by each $z \in F$, so that $a \prec_{S, F} b$. Hence, $\complement S$ is a $\preccurlyeq S, F$-class and $S$ is the union of the finite $\preccurlyeq S, F$-classes. By Lemma 6.3(ii), $S$ is the support of $\preccurlyeq S, F$.

To see that $F$ is the direction $\mathrm{f}$-cone of $\preccurlyeq S, F$, we have to show that for $z \in \mathcal{D}(S), z^{-} \preccurlyeq S, F z^{+}$if and only if $z \in F$. By construction, $z \in F$ implies $z^{-} \preccurlyeq S, F z^{+}$. Conversely, assume $z^{-} \preccurlyeq S, F z^{+}$. Then we argue as in the proof of Theorem 4.8 that $z \in F$.

Analogously to the case of general monomial preorders, we next see that each nilpotent monomial preorder contains a preorder induced by a direction f-cone.

Theorem 6.10. Let $\preccurlyeq$ be a nilpotent monomial preorder. Then $\preccurlyeq$ extends $\preccurlyeq S_{\preccurlyeq} F_{\preccurlyeq}$, the monomial preorder induced by the direction $f$-cone of $\preccurlyeq$.

Moreover, the direction cone of $\preccurlyeq S_{\preccurlyeq}, F_{\preccurlyeq}$ is $\left(S_{\preccurlyeq}, F_{\preccurlyeq}\right)$ again.

Proof. It is clear that $a \preccurlyeq S_{\preccurlyeq}, F_{\preccurlyeq} b$ implies $a \preccurlyeq b$ for any $a, b \in \mathbb{N}^{n}$; thus, $\preccurlyeq S_{\preccurlyeq}, F_{\preccurlyeq} \subseteq \preccurlyeq$ as claimed.

The second part holds by Theorem 6.9.

A Galois connection for the nilpotent case. Analogously to the general case, let us now formulate the correspondence between nilpotent monomial preorders and direction f-cones.

For a given $n \geqslant 1$, let $\mathcal{N}$ be the set of all nilpotent monomial preorders on $\mathbb{N}^{n}$ and let $\mathcal{F}$ be the set of all direction f-cones in $\mathbb{Z}^{n}$. We order $\mathcal{N}$ by set-theoretical inclusion. For $\mathcal{F}$, we make the following definition: for $(S, F),\left(S^{\prime}, F^{\prime}\right) \in \mathcal{F}$, let $(S, F) \leqslant\left(S^{\prime}, F^{\prime}\right)$ provided (i) $S \supseteq S^{\prime}$, (ii) for any $z \in F, S^{\prime}$ is closed under translations by $-z$, and (iii) $F \cap \mathcal{D}\left(S^{\prime}\right) \subseteq F^{\prime}$.

We readily check that $\leqslant$ is indeed a partial order on $\mathcal{F}$ and that the following mappings are order-preserving:

$$
\mathcal{N} \rightarrow \mathcal{F}, \preccurlyeq \mapsto\left(S_{\preccurlyeq}, F_{\preccurlyeq}\right), \quad \mathcal{F} \rightarrow \mathcal{N}, \quad(S, F) \mapsto \preccurlyeq S, F
$$

Theorems 6.9 and 6.10 indicate the result of a successive application of the two mappings: any $\preccurlyeq \in \mathcal{N}$ extends $\preccurlyeq S_{\preccurlyeq}, F_{\preccurlyeq}$, and any $(S, F) \in \mathcal{F}$ equals $\left(S_{\preccurlyeq, F}, F_{\preccurlyeq S, F}\right)$. It follows that there is a Galois connection between $\mathcal{N}$ and $\mathcal{F}$. Namely, for any $\preccurlyeq \in \mathcal{N}$ and $(S, F) \in \mathcal{F}$,

$$
\preccurlyeq S, F \subseteq \preccurlyeq \quad \text { if and only if }(S, F) \leqslant\left(S_{\preccurlyeq}, F_{\preccurlyeq}\right) \text {. }
$$


We finally apply our results to nilpotent tomonoids.

Definition 6.11. Let $(S, F) \subseteq \mathbb{N}^{n} \times \mathbb{Z}^{n}$ be such that $S$ is a finite $₫$-ideal fulfilling $(\mathrm{S})$ and $F \subseteq \mathcal{D}(S)$ fulfils (Cf1)-(Cf4). Then we call the tomonoid represented by $\preccurlyeq S, F$ an $f$-cone tomonoid.

Theorem 6.12. Each finite nilpotent p.c. tomonoid $L$ is the quotient of an f-cone tomonoid.

Proof. This holds by Theorem 6.10 and Lemma 2.8 .

Example. To illustrate Theorem 6.12, let us review the tomonoid that we considered as an example in Section 4 . Let $L$ be the 9-element tomonoid displayed in Figure 1. Note that $L$ is nilpotent. Let $\preccurlyeq$ be its representing monomial preorder; the direction f-cone $\left(S_{\preccurlyeq}, F_{\preccurlyeq}\right)$ is depicted in Figure 5.

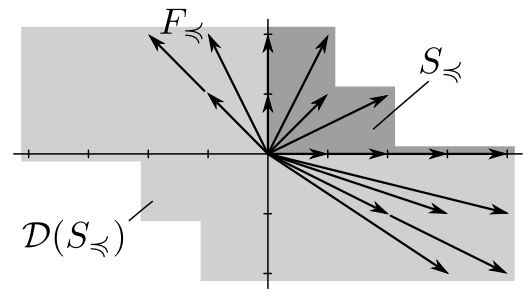

Figure 5. The tomonoid shown in Figure 1 reviewed. The support is highlighted in dark grey; its difference set consists of the support and the area highlighted in light grey. The elements of the direction f-cone are shown as vectors based at $(0,0)$.

Obviously, this representation of $L$ is more "economical" than the one discussed above. Again, we get $L$ from $\preccurlyeq S_{\preccurlyeq}, F_{\preccurlyeq}$ by making $(2,1),(1,2)$, and $(4,0)$ equivalent.

\section{Conclusion}

Each finitely generated commutative monoid $L$ is a quotient of $\mathbb{N}^{n}$. Let $L$ be endowed with a total order $\leqslant$ making it into a positive tomonoid. Pulling back $\leqslant$ to $\mathbb{N}^{n}$, we get a preorder $\preccurlyeq$ on $\mathbb{N}^{n}$, and this preorder alone determines both the congruence and the total order. In fact, the compatible, positive total preorders, called monomial preorders in this paper, correspond to finitely generated positive commutative tomonoids. In order to describe the latter, we can describe the former. This observation was the starting point of the present paper.

In order to describe a monomial preorder on $\mathbb{N}^{n}$, we have defined its direction cone as the collection of all $z \in \mathbb{Z}^{n}$ such that $a \preccurlyeq b$ whenever $b-a=z$. Direction cones are characterised by three properties reminiscent of the case of 
positive cones of partially ordered groups. By means of a direction cone alone, we cannot describe all tomonoids in question; but there is a Galois connection between the set of all monomial preorders on $\mathbb{N}^{n}$ and the set of all direction cones in $\mathbb{Z}^{n}$. A corollary of this fact is that each finitely generated positive commutative tomonoid is a quotient of a tomonoid arising from a direction cone.

We have considered in this context formally integral tomonoids. Furthermore, we have adapted our approach to the case of nilpotent tomonoids. The drawback that needs to be overcome in this latter case is the fact that a direction cone is in general infinite even if the tomonoid is finite. For this reason, we have introduced a finitary variant of direction cones and derived results in analogy to the more general case considered before.

An advance of our work is possible in many respects. Here, we have considered the finite case only under the assumption of nilpotency. General finite positive commutative tomonoids are the result of finitely many Archimedean extensions of nilpotent tomonoids, and to achieve further insight, this stepwise process should be considered.

Furthermore, we have considered one specific way of reducing an infinite direction cone to a finite object. It might be worth to take into account other possibilities. For instance, one could examine if finite subsets of $\mathbb{Z}^{n}$ generate, in some sense, direction cones. Another problem is to characterise direction cones that are minimal within the set of all direction cones.

Finally, it would be desirable to relate the present approach to other approaches that aim at a classification of tomonoids. In particular, congruences and extensions of tomonoids, which have been considered from different perspectives, e.g., in [14] and in [13], might be studied in the present context.

Acknowledgment. Open access funding provided by Johannes Kepler University Linz. I am indebted to the anonymous reviewer whose valuable comments and suggestions helped very much to improve the paper.

\section{REFERENCES}

[1] Ciabattoni, A., Metcalfe, G., Montagna, F.: Algebraic and proof-theoretic characterizations of truth stressers for MTL and its extensions. Fuzzy Sets and Systems 161, 369-389 (2010)

[2] Cox, D., Little, J., O'Shea, D.: Ideals, Varieties, and Algorithms. An Introduction to Computational Algebraic Geometry and Commutative Algebra, 3rd edn. Springer, New York (2007)

[3] Eilenberg, S., Schützenberger, M.P.: Rational sets in commutative monoids. J. Algebra 13, 173-191 (1969)

[4] Evans, K., Konikoff, M., Madden, J.J., Mathis, R., Whipple, G.: Totally ordered commutative monoids. Semigroup Forum 62, 249-278 (2001)

[5] Fuchs, L.: Partially Ordered Algebraic Systems. Pergamon, Oxford (1963)

[6] Gabovich, E.Ya.: Fully ordered semigroups and their applications. Russian Math. Surveys 31, 147-216 (1976)

[7] Grillet, P.A.: Commutative Semigroups. Kluwer, Dordrecht (2001) 
[8] Hájek, P.: Metamathematics of Fuzzy Logic. Kluwer, Dordrecht (1998)

[9] Horčík, R.: Solutions to some open problems on totally ordered monoids. J. Logic Comput. 20, 977-983 (2010)

[10] Kari, L., Thierrin, G.: Languages and compatible relations on monoids. In: Paun, Gh. (ed.) Mathematical Linguistics and Related Topics, pp. 212-220. Editura Academiei Române, Bucharest (1995)

[11] Klement, E.P., Mesiar, R., Pap, E.: Triangular Norms. Kluwer, Dordrecht (2000)

[12] Petrík, M., Sarkoci, P.: Associativity of triangular norms characterized by the geometry of their level sets. Fuzzy Sets and Systems 202, 100-109 (2012)

[13] Petrík, M., Vetterlein, Th.: The elementary extensions of finite, negative tomonoids. J. Log. Comput., to appear http://www.flll.jku.at/sites/default/files/u24/ Level-sets-for-finite-tomonoids.pdf

[14] Vetterlein, Th.: Totally ordered monoids based on triangular norms. Comm. Algebra 43, 2643-2679 (2015)

[15] Whipple, G.W.: A condition for constructing formally real monoid algebras. Algebra Universersalis 54, 53-63 (2005)

\section{ThOMAS VETTERLEIN}

Department of Knowledge-Based Mathematical Systems, Johannes Kepler University Linz, Altenberger Straße 69, 4040 Linz, Austria

e-mail: Thomas.Vetterlein@jku.at

URL: http://www.flll.jku.at/staff/vetterlein

Open Access This article is distributed under the terms of the Creative Commons Attribution 4.0 International License (http://creativecommons.org/licenses/by/4.0/), which permits unrestricted use, distribution, and reproduction in any medium, provided you give appropriate credit to the original author(s) and the source, provide a link to the Creative Commons license, and indicate if changes were made. 